\title{
Measurement of plasma electron density generated in an experiment of Laser Shock Processing, utilizing the $\mathrm{H} \alpha$-line
}

\author{
C. Moreno-Díaz , A. Alonso-Medina ,C. Colón , J.A. Porro , J.L. Ocaña
}

\begin{abstract}
A B S T R A C T
In this work we have realized plasma diagnosis produced by Laser (LPP), by means of emission spectroscopy in a Laser Shock Processing (LSP). The LSP has been proposed as an alternative technology, competitive with classical surface treatments. The ionic species present in the plasma together with electron density and its temperature provide significant indicators of the degree of surface effect of the treated material. In order to analyze these indicators, we have realized spectroscopic studies of optical emission in the laser-generated plasmas in different situations. We have worked focusing on an aluminum sample (Al2024) in air and/or in LSP conditions (water flow) a Q-switched laser of Nd:YAG $(\lambda=1.06 \mu \mathrm{m}$, $10 \mathrm{~ns}$ of pulse duration, running at $10 \mathrm{~Hz}$ repetition rate). The pulse energy was set at $2,5 \mathrm{~J}$ per pulse. The electron density has been measured using, in every case, the Stark broadening of $\mathrm{H}$ Balmer $\alpha$ line $(656.27 \mathrm{~nm})$. In the case of the air, this measure has been contrasted with the value obtained with the line of $281.62 \mathrm{~nm}$ of Al II. Special attention has been paid to the self-absorption of the spectral lines used. The measures were realized with different delay times after the pulse of the laser (1-8 $\mu \mathrm{s})$ and with a time window of $1 \mu \mathrm{s}$. In LSP the electron density obtained was between $10^{17} \mathrm{~cm}^{-3}$ for the shortest delays (4-6 $\mu \mathrm{s})$, and $10^{16} \mathrm{~cm}^{-3}$ for the greatest delays $(7,8 \mu \mathrm{s})$.
\end{abstract}

\section{Introduction}

The "Laser Shock Processing" (LSP) technique is based on focusing a high energy laser pulse ( $\mathrm{I}>10^{9} \mathrm{~W} / \mathrm{cm}^{2}, \tau<50 \mathrm{~ns}$ ) on a metallic piece what results in instant vaporization of its surface and the generation of high temperature and density plasma made of different ionized species of the elements present in the piece and in the atmosphere. The high pressure of plasma generates a shock wave which spreads into the piece affecting its mechanical features.

Since the early stages in the 70's, LSP has been consolidated as a competitive technique to improve the already mentioned features and the resistance to the corrosion of those metal surfaces used in the industry, which has turned it into a technique of practical use in industry and in engineering: stress waves generated by a giant pulsed laser were used by Fairand et al. (1972) to change in depth the microstructural and mechanical properties of 7075 aluminum. Pressures of about $10 \mathrm{GPa}$ in air environment at standard conditions were obtained by using a pulsed laser of about $10^{9} \mathrm{~W} / \mathrm{cm}^{2}$, by Fairand and Clauer (1979), confirming that the confinement of the surface with a transparent overlay provides an effective method of generating high-amplitude laser induced stress waves in the target material. The Laser Shock Processing of water-immersed material was developed, by Sano et al. (1997), for improving the residual surface stress of metal.

There are works focused on finding the best application conditions of the technique. Fabbro et al. (1990) established the three fundamental stages of evolution of plasma in an experiment of laser-produced plasma in confined geometry. In that study it indicated that during a time period of about twice the duration of the laser pulse is detected on the target high pressure. It is further noted that for later times, and while the plasma cools adiabatically, the pressure decreases and is too small to produce plastic deformation in the material. Berthe et al. (1997) indicated that this decrease in pressure was due to parasitic effects of the dielectric breakdown of the water when the laser irradiance exceeds $10 \mathrm{GW} / \mathrm{cm}^{2}$. Ocaña et al. (2004) presented a summary of different experimental results obtained from the LSP experiments, with some conclusions for the assessment of LSP technology as a profitable method for the extension of fatigue life in critical heavy duty components.

There are also works aimed to study theoretically some of the aspects involved in the physical processes present in LSP. A theoretical model was presented by Ocaña et al. (2000), in order to found 


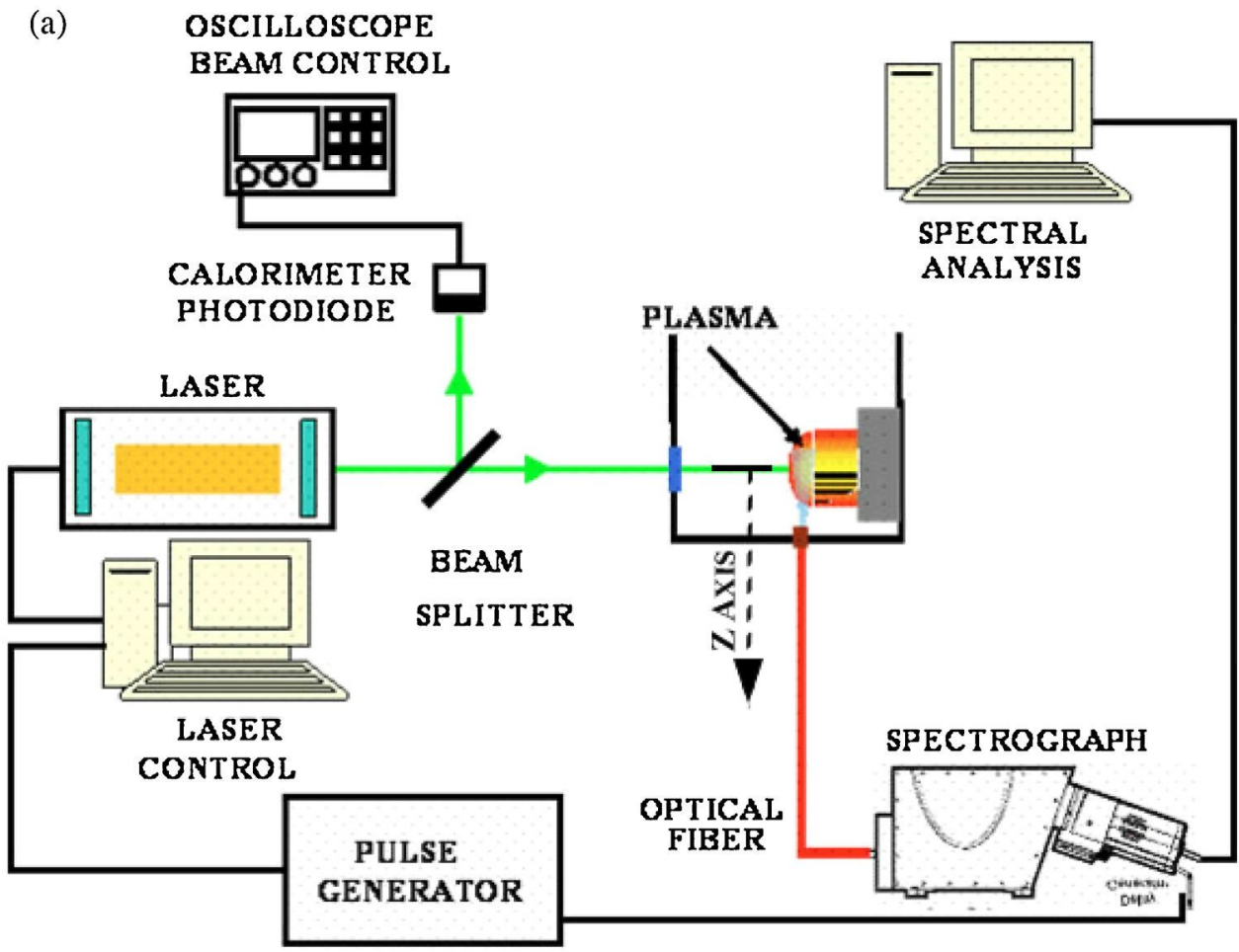

(b)

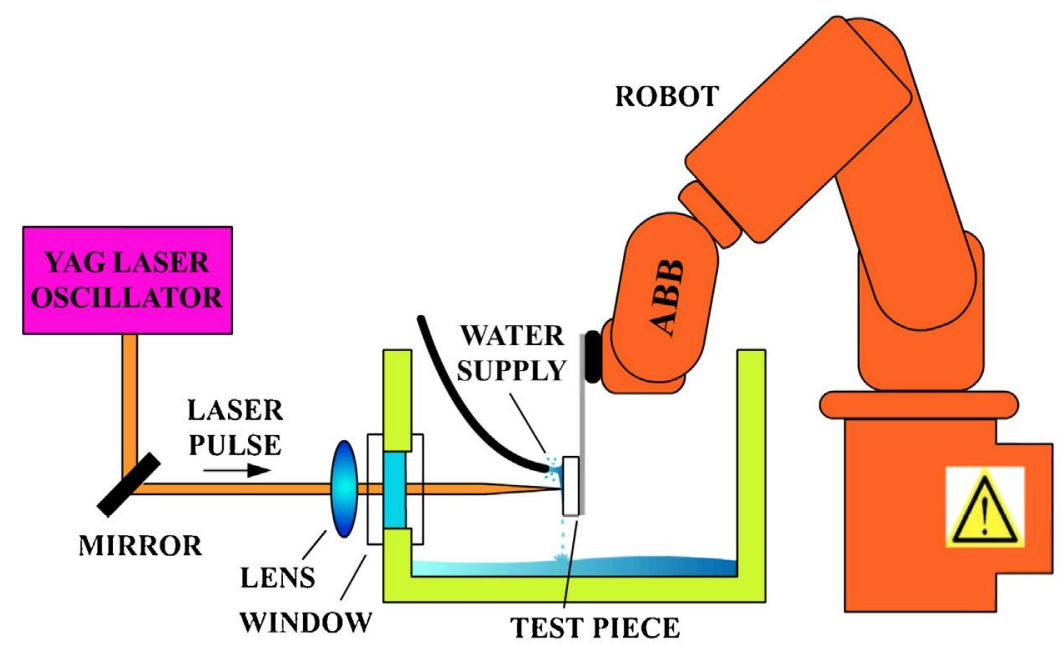

Fig. 1. (a) Experimental setup in an irradiation LSP experiment. (b) Detail indicating the relative movement of the sample to the measuring system.

the practical criteria to optimization of process. This model was later refined by Morales et al. (2008). A self closed thermal model for laser peening under water confinement regime was presented by $\mathrm{Wu}$ and Shin (2005). In this work the authors present several comparisons between their model and the experimental results for the peak pressure claiming that their model predicts number densities of electron that agree reasonable well with the experimental results. Nevertheless, theoretical works are far from being complete, since there is an important gap at predicting which the best conditions of application of LSP will be when trying to apply the technique to materials different from those previously studied. This is partly due to the physical processes involved.

One of the physical processes that should be deeply studied consists of the interaction between plasma and the surface of the material and how it can be affected by mechanisms different from the mere mechanical interaction with the shock wave. It is evi- dent that the ionized species in the plasma will cause chemical attack on the surface of the sample. The presence of hydrogen in the plasma is a test of these chemical mechanisms. To study such process, it is necessary to know the properties of plasma generated by the interaction between the laser and the metallic surface. From the knowledge of electron density and electron temperature of the plasma it is possible to establish a balance of the relative presence of different ions in the plasma.

This type of plasma studies has been realized for years and they are known as Laser Induced Breakdown Spectroscopy (LIBS). A detailed study of laser induced plasmas and its applications were submitted by Radziemski and Cremers (1989). A earlier compilation of the applications of plasmas produced by laser for spectroscopic techniques was presented by Noll (2012). A more recent collection of experiments and applications of the LIBS technique were presented by Musazzi and Perini (2014). 
LIBS is one of the most versatile methods which can be applied in multiple fields of science. Cremers and Chinni (2009), present an overview of the LIBS method, discussing its many advantages and some important limitations as an analytical method and how these relate to potential applications. It also outstands as an analytical technique in different industrial applications (Noll et al., 2008).

LIBS is based on the spectroscopic study of the emission of the present species in the plasma, and the advantage consists of obtaining its parameters and has been used by these authors in several works. Before, we have obtained temperature, electron density, atomic parameters and Stark broadening parameters in different laser induced plasma as: several parameter in aluminum plasma (Colón et al., 1993); transition probabilities of Pb III in lead plasma (Colón et al., 1999); Stark widths of spectral lines of Pb II in lead plasma (Colón and Alonso-Medina, 2006); transition probabilities of $\mathrm{Pb} \mathrm{Sn}$ II in tin plasma (Alonso-Medina et al., 2003); Stark widths of several $\mathrm{Pb}$ III spectral lines in lead plasma (Alonso-Medina and Colón, 2007); Stark widths of several Sn I and Sn II spectral lines in tin plasma (Alonso-Medina and Colón, 2008); transition probabilities of Sn I in tin-lead plasma (Alonso-Medina, 2010) and recently the transition probabilities of Ca I in calcium-lead plasma (AlonsoMedina, 2014).

There are some thermo-fluid-dynamic models, which try and predict the temperature, the density of electrons of plasma and the pressure of the shock wave, such as the HELIOS package. This package is a "radiation-hydrodinamics 1-D code" used to simulate the dynamics of evolution of plasma (Lyon and Johnson, 1992). This code has proved its capacity to predict the pressure of the shock wave. However, this model fails spectacularly when diagnosing the plasma, because in case of working without water (LIBS experiments) it predicts lower temperatures than those measured, one order of magnitude lower than the experimentally measured ones, and electron densities much higher than the ones found. Possibly this code fails by not taking into account the different chemical mechanisms that take place in the plasma, and between the plasma and the target material. Therefore, in case of working in water flow,

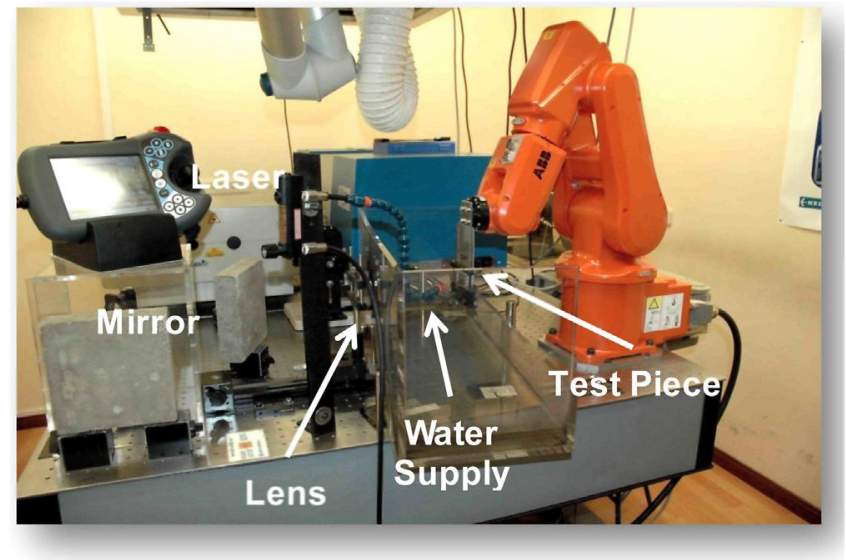

Fig. 2. Photographic view of the LSP irradiation experimental setup.

this makes the measures of the parameters of plasma estimated by the HELIOS package do not look reliable a priori.

The difference, between the study presented in this paper and the studies mentioned, depends on the shielding the flow water applies on the emission of every present species in the plasma with a exception: the Hydrogen emission. Historically, the $\mathrm{H} \alpha$-line was successfully used to diagnose several kinds of plasmas. The earlier detection of $\mathrm{H} \alpha$-line was made by Bogen and Lie (1980) and was used for several measurements in vacuum by Ashkenazy et al. (1991). The same line was used to determine the electron density in a laser produced plasma in different conditions: in air (humid air by El Sherbini et al., 2006 and atmospheric air by De Giacomo et al., 2008), in aqueous medium (Escarguel et al., 2000) and in a solid target in a liquid medium (water) (titanium target and sea water by De Giacomo et al., 2004).

In this work, by using the classical LIBS technique, the plasma density has been measured using $\mathrm{H} \alpha$-line. $\mathrm{H} \alpha$-line is a well isolated line, it gives large signal to background ratio, it lasts a long time after

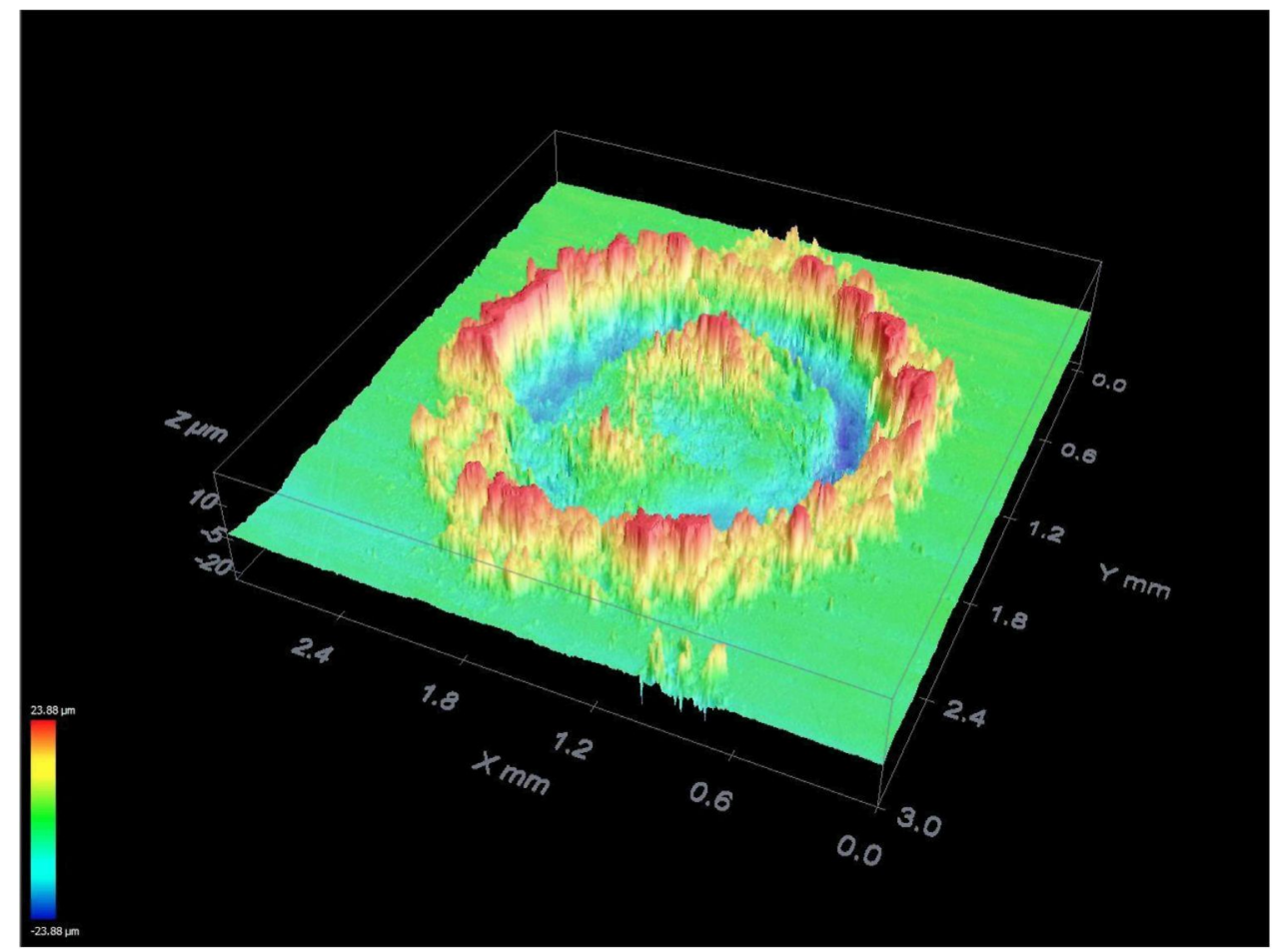

Fig. 3. Image obtained via scanning confocal microscopy. A typical surface changed after a LSP experiment. 
the termination of the laser (up to $10 \mu$ s), its Stark width is relatively large and does not exhibit self-absorption. This procedure has been realized in experiments with a sample of aluminum (Al2024) in every air, wet air and water flow. Also, where possible (air and wet air) other species have been used ( $\mathrm{Al} \mathrm{II,} \mathrm{Cu} \mathrm{I,} \mathrm{Mg} \mathrm{I} \mathrm{and} \mathrm{Mg} \mathrm{II)} \mathrm{in}$ an attempt to estimate the plasma temperature. All measures were realized with different delay times after the laser (4-8 $\mu$ s) and with a measure time window of $1 \mu \mathrm{s}$.

In this paper we provide a method of experimental measurement of plasma parameters in LSP conditions, so they can be compared with the predictions of theoretical models and they can be tuned. That is why our study is carried out for long time, when the plasma is adiabatic cooling, the $\mathrm{H} \alpha$-line appears and we can measure using spectroscopic techniques.

\section{Procedure and experimental setup}

When a target is irradiated by an intense $\left(>1 \mathrm{GW} / \mathrm{cm}^{2}\right)$ laser pulse, the surface layer vaporizes into a high temperature and pressure $(1-10 \mathrm{GPa})$ plasma. The plasma induces shock waves during expansion from the irradiated surface and mechanical impulses are transferred to the target. If the plasma is confined by water or other medium, the shock pressure into the material can be magnified by a factor of 5 or more compared with the open air condition. These pressures are well above the yield stress of most metals, thus plastic deformation can be induced. As a result, if the peak shock pressure is over the Hugoniot elastic limit (HEL) of the target material for a sufficient time, compressive residual stress distribution in the surface of the irradiated volume can be induced as a consequence of the plastic. Water is usually used as a confining condition.

The experimental device is analogous to the one used by those authors in the LIBS experiments [12-17]. But we have to take into account the confining presence of water in the experiments for the plasma diagnosis in LSP conditions. A schematic diagram of the experimental set-up is shown in Fig. 1.

A Q-switched Nd-YAG laser (Quanta-Ray PRO 350 de SpectraPhysics) was used at the fundamental wavelength of $1.06 \mu \mathrm{m}$. The energy per pulse ( $10 \mathrm{~ns}$ width) was measured at the target surface and was found to be $2.5 \mathrm{~J}$. An autofocus system with a simple lens $(f=20 \mathrm{~cm}$, biconvex $)$ focuses the laser light on the sample. There is also a vessel a water supply system via hose connection, which offers a confining environment in permanent circulation and a programmable 3D positioning system, the sample is over it, which allows us to control the distances between the lens and the sample and thus the spot size. A photographic view can be seen in Fig. 2.

The target used is a certified aluminum based alloy (A12024) with a $\mathrm{Mg}$ content of $\sim 1.5 \%$. In our experiment, the spot size, measured at the target surface, was a circle of diameter $\sim 2 \mathrm{~mm}$. A typical surface changed after a LSP experiment can be seen in Fig. 3. It deduces that the irradiance of laser was around $10 \mathrm{GW} / \mathrm{cm}^{2}$

The laser energy was monitored using a calibrated powermeter Ophir (F100A-HE-RP), utilizing the reflected part from a quartz beam splitter. The reflectance and absorption of the splitter were measured at the laser wavelength and found to be $5 \%$ and $3 \%$, respectively. Emission spectra were acquired using a spectrograph (Horiba Jobin Ybon FHR1000) equipped with a CCD camera (Andor, model iStar 334T). This camera's CCD is bidimensional, $1024 \times 1024$ pixels ( $26 \times 26 \mu \mathrm{m}^{2}$ size per pixel) which has allowed us to study the plasma spatially. The camera is time controlled both in gate and delay with a maximum resolution of $100 \mathrm{~ns}$. The spectrometer is equipped with a diffraction grating of 1800 groves $/ \mathrm{mm}$ and covers a wavelength region from 200 to $700 \mathrm{~nm}$. The light emitted is transferred to the spectrograph using a quartz fiber cable of $0.5 \mathrm{~mm}$ diameter. The data was taken at the surface of the target up to $2 \mathrm{~mm}$ normal to the target surface (where the signal/noise ratio (a)

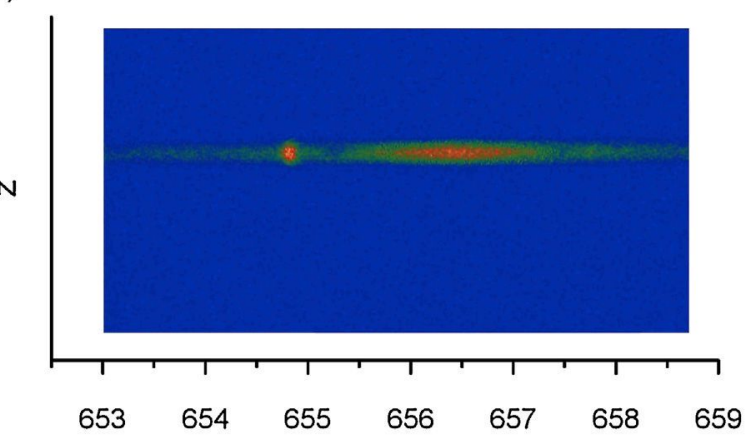

(b)

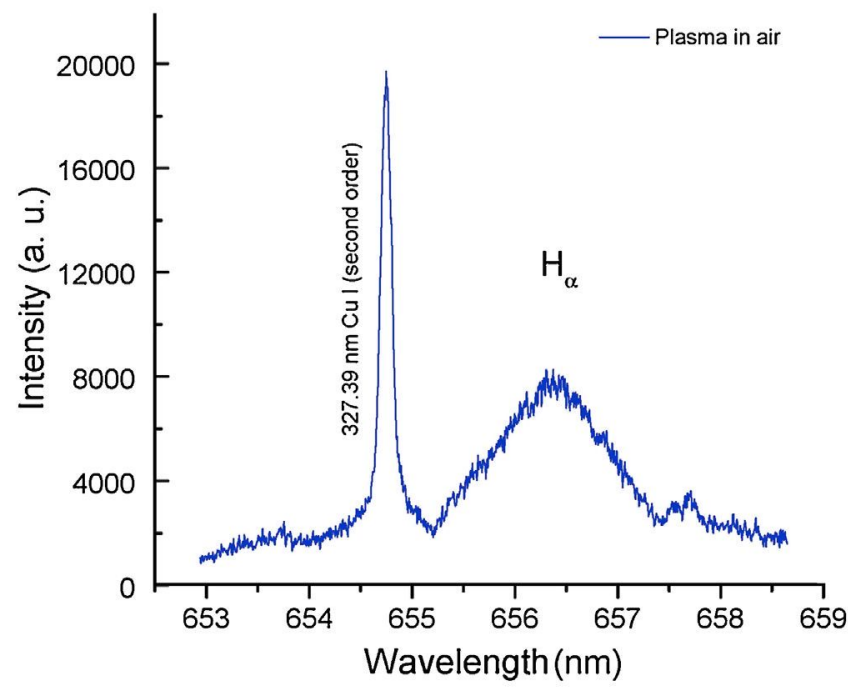

Fig. 4. (a) Spectrally resolved image of the plasma at $5 \mu$ s after laser pulse with a wavelength range $653-659 \mathrm{~nm}$ and (b) Spectra extracted from this image of the $\mathrm{H} \alpha$-line in air.

was the best found). The wavelength scale was calibrated with a low pressure Hg-lamp (Oriel). The instrumental bandwidth (measured with the Hg-lamp) was found on the average to be $0.03 \pm 0.01 \mathrm{~nm}$ over the different wavelength regions. Also, we have calibrated the spectral response of the system (efficiency) between a 200 and $700 \mathrm{~nm}$ wavelength range by means of previously calibrated lamps which are a deuterium lamp used in the range of $190-400 \mathrm{~nm}$ and a tungsten lamp for the 350-700 nm range.

As an example of the spatial resolution of the images obtained, in Fig. $4 \mathrm{a}$ and b, we can see images of the plasma trace at $5 \mu \mathrm{s}$ after laser pulse whose measures have been realized in air.

The obtained spectra were stored in a computer for further analysis The radial distribution of the signal was obtained from the integrated data, obtained directly, using Abel inversion process. The spectra line analysis was performed by fitting the observed line shapes to numerically generated Voigt profiles, which consists of the convolution obtained with the Lorentz and Gauss profiles. For the plasma diagnostic, the area under each line profile from the aforementioned fitting represents the relative intensity. The identification of lines was realized using the NIST Tables (Kramida et al., 2014). The self-absorption analysis of the used spectral lines received special attention.

\section{Results and discussion}

We have obtained the plasma emission spectra in the range of $200-700 \mathrm{~nm}$ at different times after the laser pulse $(1-8 \mu \mathrm{s})$. The 


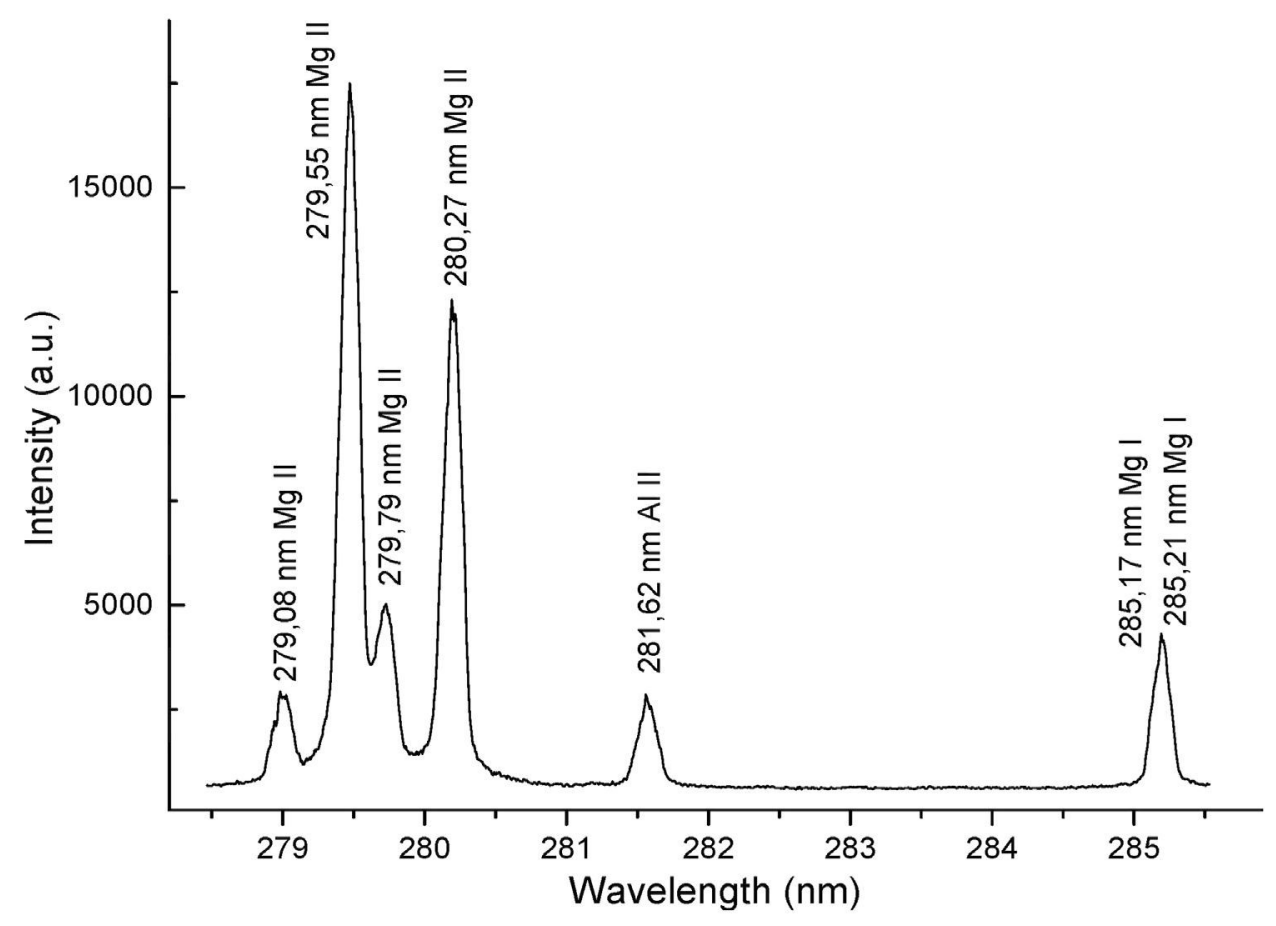

Fig. 5. Typical spectra for $278-286 \mathrm{~nm}$ in air at $5 \mu$ s after laser pulse.

spectra were obtained in air, in air with a wet sample and also in LSP conditions (flow water over the sample). The main characteristic is that in the first two cases the spectra presents the emission of several atoms and species ( Al I, Al II, Mg I, Mg II, Cu I, Cu II, H I. . .). A typical section of the spectra in these two first cases can be seen in Fig. 5, and as shown, it is analogous to the one presented by other authors in works realized in similar conditions (El Sherbini et al., 2012).

In Fig. 6a, you can see the spectra emitted by the plasma in the emission area of $\mathrm{H} \alpha$-line at different times and several conditions (air and LSP) after the laser pulse. As can be seen a long time in all cases appears the $\mathrm{H} \alpha$-line of Hydrogen. The hydrogen emission from metallic samples in air has already been found by other authors and has been widely discussed in the literature. Also, in LSP conditions the hydrogen $\mathrm{H} \alpha$-line, appears clearly at $4 \mu$ s after the laser pulse. For example, In Fig. 6 b, this very area is presented at $5 \mu \mathrm{s}$ after the laser pulse in different conditions: Plasma in air, plasma in air with a wet sample and plasma in LSP conditions (water flow).

As shown, the H $\alpha$-line and the line $327.39 \mathrm{~nm}$ (second order) of $\mathrm{Cu}$ I only appear at long times ( $4 \mu \mathrm{s}$ after the laser pulse). Furthermore, in the presence of water in LSP conditions, the Cu I line disappears due to the shielding above mentioned. In air, and with wet target, traces of the emission of $\mathrm{Cu}$ I are still observed. Although expected the water should affect the plasma conditions, we have found, that at $5 \mu \mathrm{s}$ after the laser pulse, the profile and the position of the $\mathrm{H} \alpha$-line has remained unchanged indicating that at that time the electron density and the electron temperature plasma are identical within the experimental uncertainties.

As previously mentioned, the lines of interest in this work have been studied while fitting numerically Voigt profile in order to deduce its area and the Lorentz component of the width as shown in Fig. 7a, where the red solid curve is the fit of the experimental data (black color).

Fig. 7b shows the fittings obtained for the spectra measured at different distances from the plasma axis in the area of $\mathrm{H} \alpha$-line. As you can see, except for the differences linked to the lower intensity of the lines while going farther from the plasma axis, the profiles of $\mathrm{H} \alpha$-line (position of maximum and Full Width at Half
Maximum-FWHM) are similar within the experimental uncertainties. Similar result has been found in the area of $281 \mathrm{~nm}$ in case of plasma in air. As an example, Fig. $8 \mathrm{a}$ and $\mathrm{b}$ present the results obtained. Owing to this, when using the original data integrated to do Abel's inversion, the results of this treatment give us results in the profile of the lines which do not differ within the experimental uncertainties of the results obtained with the original data. These are the integrated data which have been used along the whole subsequent study.

Once this first step has been done, in order to estimate electrons temperature and density, we have studied possible self-absorptions of the lines.

\subsection{Self-Absorption analysis}

The self-absorption (SA) coefficient focused on line $\left(\lambda_{0}\right)$ results from the expression (Lochte-Holtgreven, 1968; Griem, 1974; Konjevic, 1999).

$\mathrm{SA}=\frac{I\left(\lambda_{0}\right)}{I_{0}\left(\lambda_{0}\right)}=\frac{\left[1-e^{-\kappa\left(\lambda_{0}\right) \ell}\right]}{\left[\mathcal{K}\left(\lambda_{0}\right) \ell\right]}$

where $I\left(\lambda_{0}\right)$ is the relative intensity subject to self-absorption, $I_{0}\left(\lambda_{0}\right)$ is the same as the former, but without self-absorption, and $\kappa\left(\lambda_{0}\right) \ell=\tau\left(\lambda_{0}\right)$ is the plasma optical path until the center of the line.

On the other hand, as the self-absorption process affects FWHM $\Delta \lambda_{0}$ the SA in terms of ratio of the Lorentzian is given by El Sherbini et al. (2005):

$\mathrm{SA}=\left(\frac{\Delta \lambda}{\Delta \lambda_{0}}\right)^{1 / \alpha}=\left(\frac{N_{\mathrm{e}}(\text { line })}{N_{\mathrm{e}}^{*}}\right)^{1 / \alpha}$

where $\alpha=-0.56, \Delta \lambda_{0}$ is the FWHM of the Lorentzian component without self absorption, $\Delta \lambda$ is the FWHM of the Lorentzian component distorted for the self absorption, $N_{e}$ is the apparent electron density deduced from the self absorption distorted line and $N_{\mathrm{e}}{ }^{*}$ is the real electron density. 

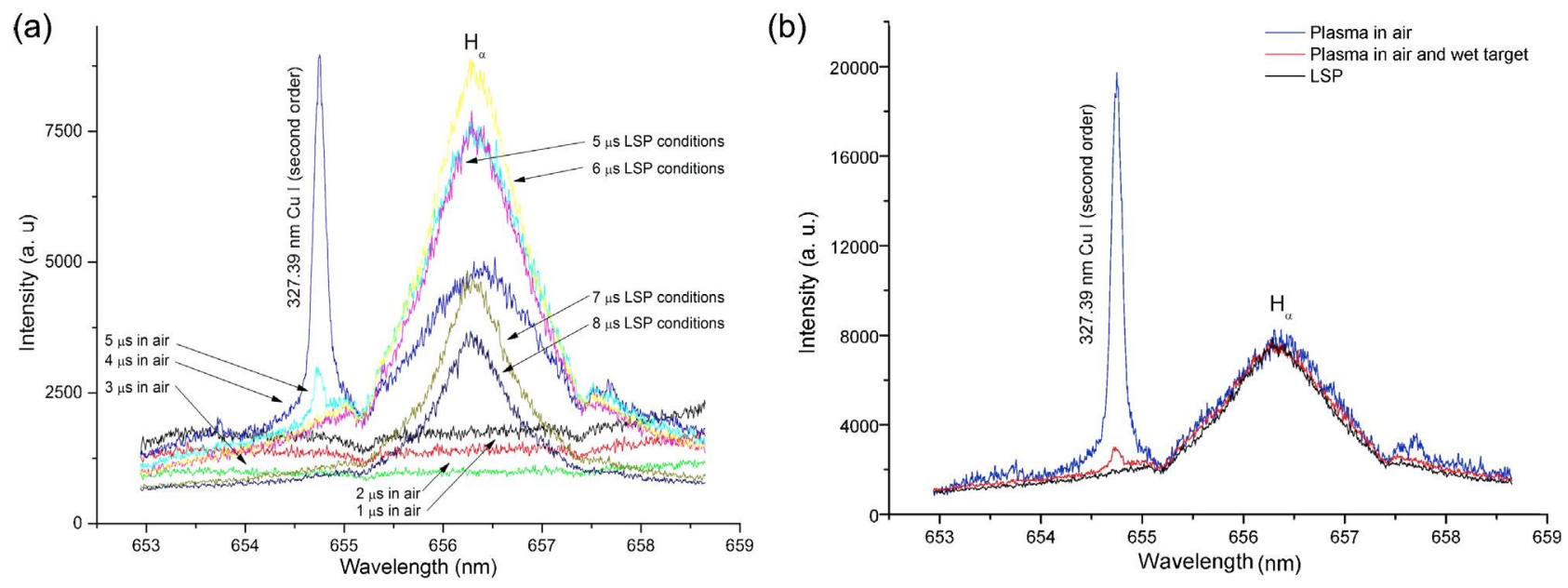

Fig. 6. (a) $\mathrm{H} \alpha$-line emission at different delay times from laser pulse and (b) $\mathrm{H} \alpha$-line emission at $5 \mu$ s after laser pulse.

(a)

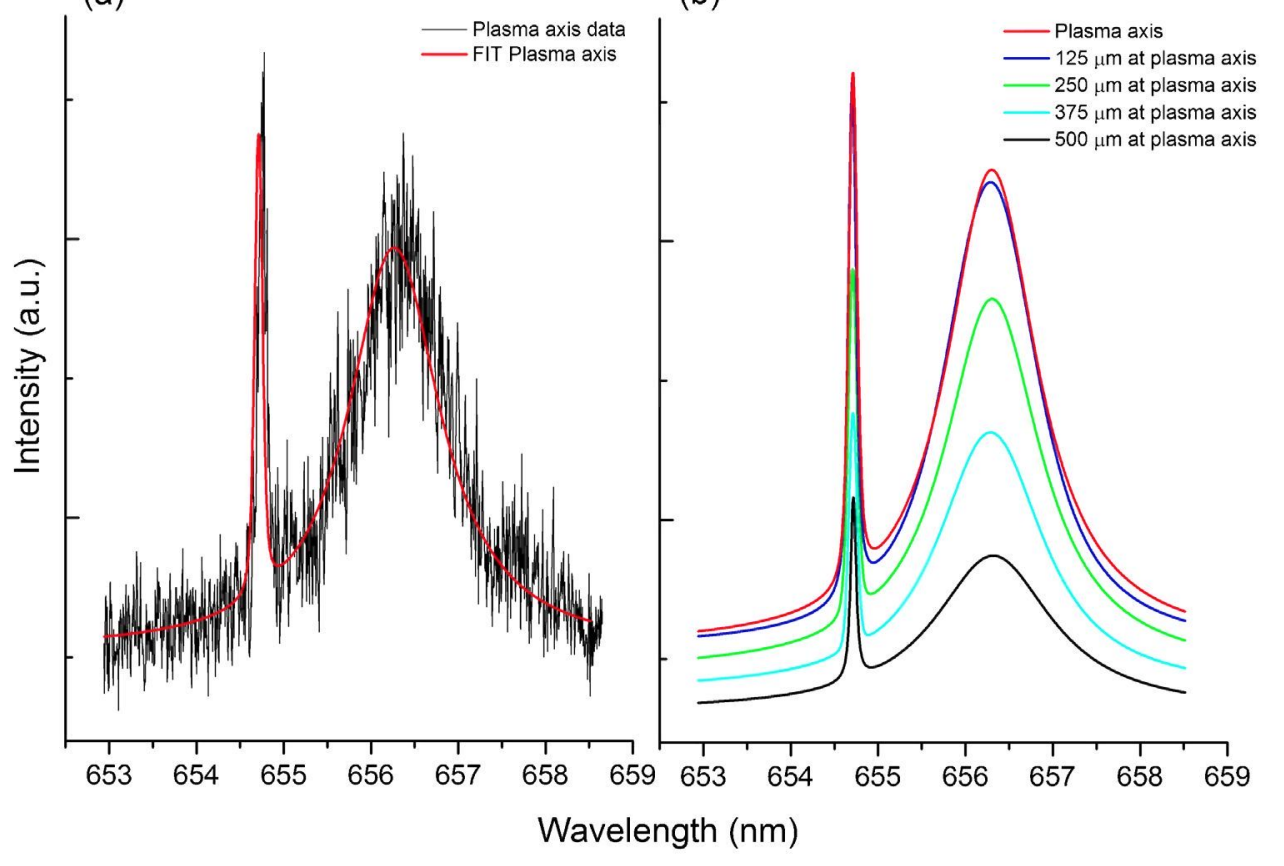

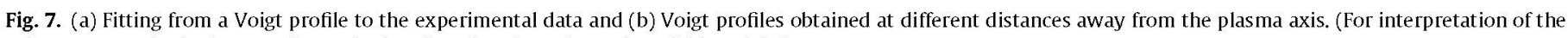
references to color in the text, the reader is referred to the web version of this article.)

In this work, you can observe that $\mathrm{H} \alpha$-line of Hydrogen is present in every spectrum obtained from different conditions, and it is the only one in the water flow case. In addition, in every case $\mathrm{H} \alpha$-line is perfectly symmetrical and does not present decrease in the center, this confirms self-absorption absence for this line (as shown in Fig. 6b). This fact had already been emphasized by El Sherbini et al. (2006). Besides, according to Griem, (1974), in plasmas with electron densities over $10^{17} \mathrm{~cm}^{-3}$ using this line is advantageous over using $\mathrm{H} \beta$-line whose broadening can hardly be discerned from the ground.

Due to all this, $\mathrm{H} \alpha$-line is going to be used to estimate the real density of electrons in LSP experiments. Such a line will be also used to calculate SA coefficients of spectral lines of different species which can be of interest in successive analysis in this work.

In Fig. 6b, we can also see that in all three cases presented ( $5 \mu \mathrm{s}$ after laser pulse) $\mathrm{H} \alpha$-line does not widen or shift. This means that the electron density and the temperature are the same within the experimental uncertainties in both three cases regardless of the amount of water.

\subsection{Electron number density determination}

In order to obtain the electron density from the $\mathrm{H} \alpha$-line, we have used the following expression, Ashkenazy et al., 1991:

$N_{\mathrm{e}}=8.02 \times 10^{12}\left(\frac{\Delta \lambda_{1 / 2}}{\alpha_{1 / 2}}\right)^{3 / 2} \mathrm{~cm}^{-3}$

where $\Delta \lambda_{1 / 2}$ is the FWHM of the line in $\AA$, and $\alpha_{1 / 2}$ is half the width of the reduced Stark profiles in $\AA$ and it is a weak function of electron density and temperature through the ion-ion correlation and Debye-shielding correction and the velocity dependence of the impact broadening. Precise values of $\left(\alpha_{1 / 2}\right)$ for the Balmer series can be found in (Griem, 1974; Kepple and Griem, 1968). 


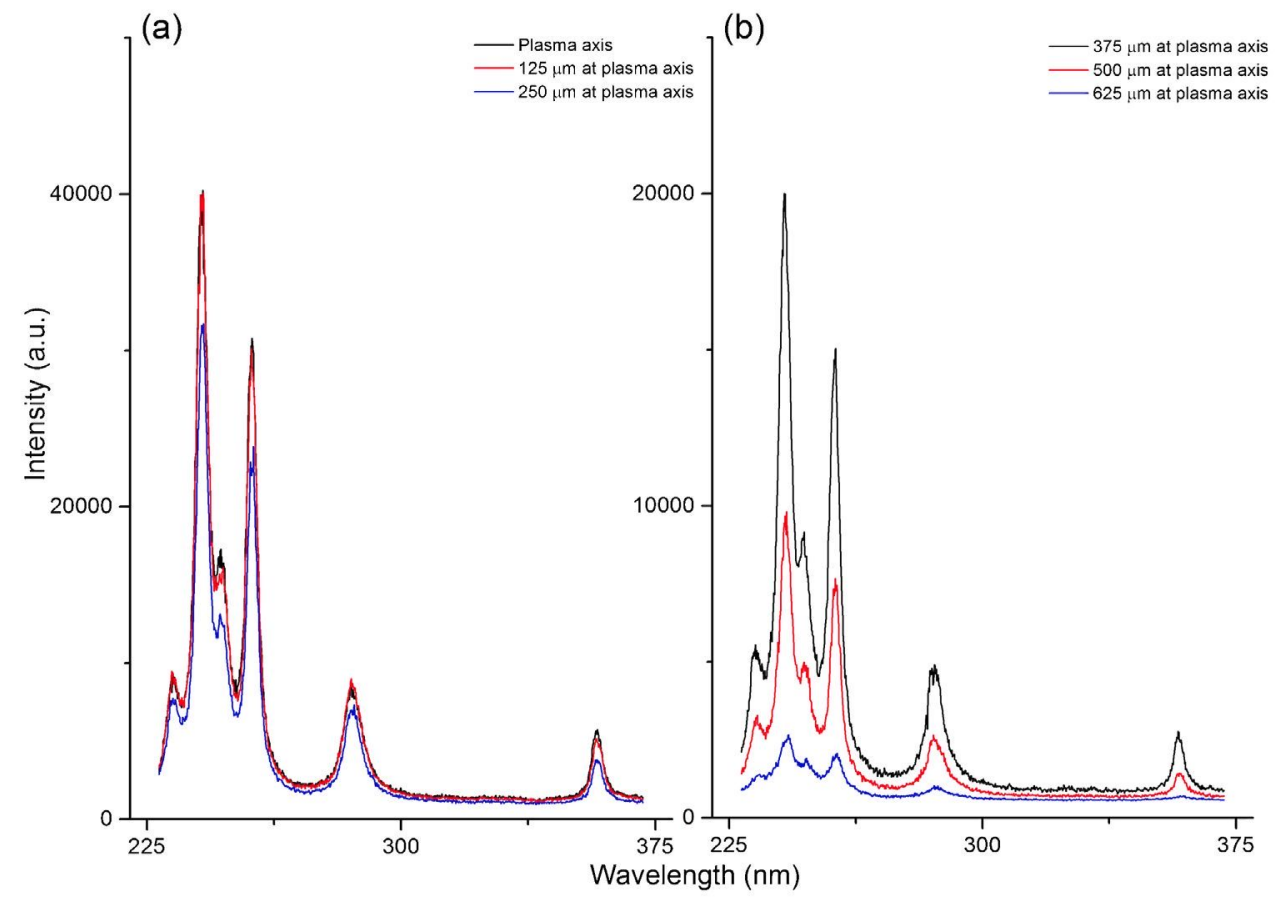

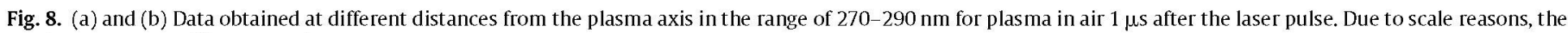
results are presented in two sections.

As can be seen in the already mentioned references, $\alpha 1 / 2$ parameter is little sensitive to temperature. It is well known that for plasmas in air in experimental conditions analog to those in this work, temperature will be between 7000 and 13,000 K (El Sherbini et al., 2006; De Giacomo et al., 2008). In this work we have used the parameter given by Griem at a temperature of $10,000 \mathrm{~K}$. This assumption will be justified later.

In order to calculate the Stark contribution, $\Delta \lambda_{1 / 2}$, to the measured lines width, a fitting of the lines to the Voigt profile was carried out. This has allowed us to find the Lorentz component where we discounted the Lorentz part of the instrumental profile. In addition, we have taken into account the different broadening mechanisms. The most important mechanism, the Doppler broadening, was discounted to dispense with the Gauss profile of the Voigt. In our conditions, other broadening mechanisms are irrelevant.

In addition, with this expression, we have estimated the electron density profile in the plasma axis ( $z$ axis, see Fig. $1 a$ ) verifying again the possibility of working just with integrated values. The spatially resolved profiles of the electron density along the $z$ axis at $5 \mu \mathrm{s}$ delay from the laser pulse (in LSP conditions) are shown in Fig. 9. The uncertainty present in this figure is underestimated: includes the error on the value of FWHM determined by fitting (3-5\%) and does not include the possible error provided by the approximation of the coefficient $\alpha_{1 / 2}$. The result obtained with integrated values for $5 \mu$ s (in LSP conditions) as shown hereafter in Table 1 is $1.36 \times 10^{17} \mathrm{~cm}^{-3}$. This result, obtained from the integrated values, is a correct supposition, as supports Fig. 9. For the rest of the delay times, we have only worked with integrated values.

The results achieved through this procedure are shown in Table 1. In the first column we have different delay times from the laser pulse. In the second column, the broadening of those studied conditions is displayed. At last, in the third column the corresponding electron densities are shown. It is well known that for electron densities over $30 \times 10^{17} \mathrm{~cm}^{-3}$, other authors (Ashkenazy et al., 1991) have seen how self-absorption processes start to appear. Therefore, the electron densities obtained in our work are coherent with our former explanation: $\mathrm{H} \alpha$-line does not present self-absorption.

Attempts have been made to verify the electron density in the case of plasma in air using line $281.62 \mathrm{~nm}$ of Al II which is usually used in aluminum plasmas. Then, we have used Griem expression:

$N_{\mathrm{e}}=\frac{\Delta \lambda_{1 / 2}}{\omega_{\text {ref }}} \times 10^{16}$

where $\Delta \lambda_{1 / 2}$ is the FWHM of the line in $\AA$, and $\omega_{\text {ref }}$ is the impact broadening factor (normalized at $10^{16} \mathrm{~cm}^{-3}$ ) and $N_{\mathrm{e}}$ is the electron number density. By using the impact broadening factor measured by Colón et al. (1993), we obtain a value $\sim 4 \times 10^{17} \mathrm{~cm}^{-3}$. This value is about twice higher that obtained with the $\mathrm{H} \alpha$-line. This discrepancy seems to indicate that the lines of Al II are self-absorbed what is not advisable to be used in our case. As conditions of selfabsorption of lines of different species are different, the lines of Al II may be self-absorbed, although it is not the case of $\mathrm{H} \alpha$-line. The difference between the electron densities obtained with the $\mathrm{H} \alpha$-line and electron densities obtained with the lines of Al II, allow us, as shown below, correct the self-absorption of the intensity of these lines.

\subsection{Electron temperature}

Electron temperature could be estimated through the Boltzmann plot method under the assumption that the plasma is in local thermodynamic equilibrium (LTE) (Griem, 1974; Griem, 1997) by using:

$$
\begin{aligned}
& I_{i j}^{\lambda}=\frac{A_{i j} g_{i}}{U(T)} N \exp \left(\frac{-E_{i}}{k T}\right) \\
& \ln \left(\frac{I_{i j}^{\lambda}}{A_{i j} g_{i}}\right)=\ln \left(\frac{N}{U(T)}\right)-\frac{E_{i}}{k T}
\end{aligned}
$$

for a transition from a higher state $i$ to a lower state $j, I_{i j}{ }^{\lambda}$ is the integrated measured integral line intensity in counts per second, $A_{i j}$ 


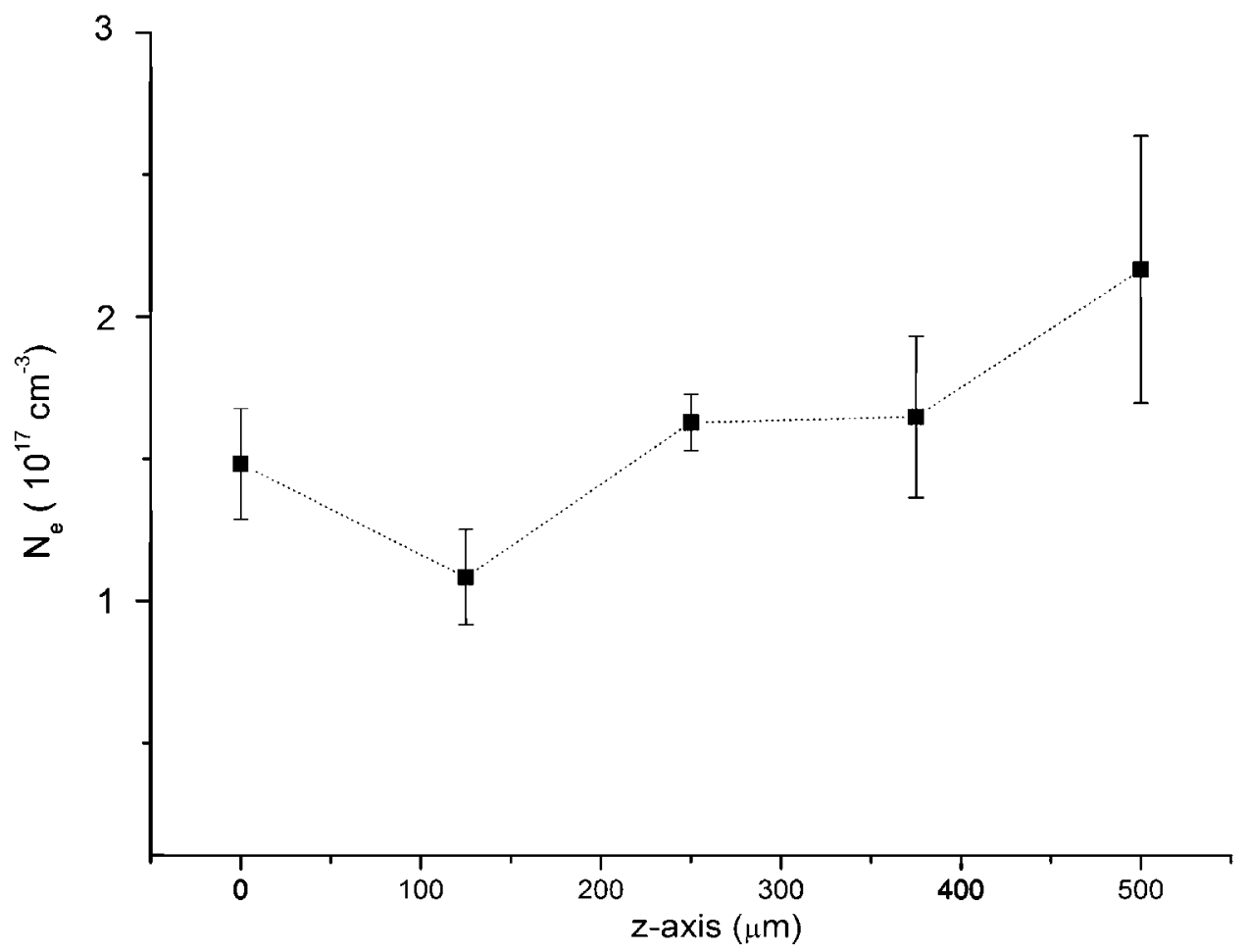

Fig. 9. Space-resolved of the plasma electron density measurement from the Stark broadening of H Balmer $\alpha$ line in LSP conditions.

Table 1

Density of plasma electrons studied in this work in different (air, wet target and LSP) and at different times.

\begin{tabular}{|c|c|c|c|c|c|c|}
\hline \multirow[t]{2}{*}{ Delay time after laser pulse, $\tau(\mu s)$} & \multicolumn{3}{|c|}{$\operatorname{FWHM}\left(\Delta \lambda_{1 / 2}\right) \AA$} & \multicolumn{3}{|c|}{$N_{\mathrm{e}}\left(10^{17} \mathrm{~cm}^{-3}\right)$} \\
\hline & Air & Wet target & LSP & Air & Wet target & LSP \\
\hline 4 & $14.33 \pm 3 \%$ & $14.53 \pm 3 \%$ & & $1.72 \pm 3 \%$ & $1.75 \pm 3 \%$ & \\
\hline 5 & $13.11 \pm 3 \%$ & $13.18 \pm 5 \%$ & $12.30 \pm 4 \%$ & $1.50 \pm 3 \%$ & $1.51 \pm 5 \%$ & $1.36 \pm 4 \%$ \\
\hline 6 & & & $11.79 \pm 3 \%$ & & & $1.28 \pm 3 \%$ \\
\hline 7 & & & $9.13 \pm 3 \%$ & & & $0.87 \pm 3 \%$ \\
\hline 8 & & & $9.06 \pm 3 \%$ & & & $0.86 \pm 3 \%$ \\
\hline
\end{tabular}

is the transition probability, $\lambda$ is the wavelength of the transition, $E_{i}$ is the excited level energy and $g_{i}$ is the energy and statistical weight of level $i, U(T)$ is the atomic species partition function, $N$ is the total density of emitting atoms, $k$ is the Boltzmann constant and $T$ is the temperature in $\mathrm{K}$. If we were to plot $\ln \left(I_{i j}{ }^{\lambda} / A_{\mathrm{ij}} g_{i}\right)$ vs. $E_{i}$, for lines of known transition probability (Boltzmann plot), the resulting straight line would have a slope $-1 / k T$, and therefore the temperature could be obtained without having to know the total density of atoms or the atomic species partition function.

In LSP experiments, temperature cannot be directly determined through this procedure because as already mentioned, the presence of flow water shields, the emission of every species but hydrogen. However, it will be estimated with the results obtained from an experiment in air in identical conditions as the LSP experiment. As we mentioned above, the profile and the position $\mathrm{H} \alpha$-line is identical in both cases, Fig. 6b, what, as mentioned before, allows us to extrapolate the temperatures from one to the other. Therefore, temperature in air has been estimated. In order to estimate the temperature in air, we have used, as usual, the spectra in the range of 270-290 nm, where several lines of Mg II appear. Spectral lines used in the Boltzmann plot along with the transition probabilities and the energies corresponding to different starting levels, are presented in Table 2 . Also, Table 2 shows the $\omega_{\text {ref }}$ (impact broadening factors) measured by different authors and necessary for the line self-absorption corrections.

To estimate absorption, we have adjusted Voigt profiles to such lines to determine its Lorentz broadening and to get, through equation (1) apparent electron densities $\left(N_{\mathrm{e}}{ }^{*}\right)$. Thus, and compared with the electron density and the $\mathrm{H} \alpha$-line $\left(N_{\mathrm{e}}\right)$, we will get, using equation (2), the corresponding self-absorption coefficients (SA) in every line. Finally, with these coefficients, we will estimate the relative intensity in the limit of nule self-absorption (negligible self-absorption), $I_{0}\left(\lambda_{0}\right)$.

In this work, when there are $5 \mu$ s after laser pulse, the result (using values $I\left(\lambda_{0}\right)$ ) before correcting the lines selfabsorption has been $19,400 \pm 1000 \mathrm{~K}$ for $\Delta E=4.442 \mathrm{eV}$. Once the self-absorption has been corrected (using $I_{0}\left(\lambda_{0}\right)$ ) we have obtained $10,900 \pm 1000 \mathrm{~K}$. These results can be seen in Fig. 10. In our calculations the uncertainty present includes the statistical error on the fitting in the temperature value and the statistical uncertainty in the intensities $(\sim 3 \%)$.

This procedure has been repeated for different times (4-8 $\mu \mathrm{s}$ ) obtaining temperatures between 12,000 and $9000 \mathrm{~K}$. These results are consistent choosing $\alpha_{1 / 2}$ parameter which is used to determine the electron density since $\mathrm{H} \alpha$-line. These values, obtained in air, are considered to be an estimate of the LSP case. 
Table 2

Parameters of $\mathrm{Mg}$ II spectral lines used for electron temperature determination and for the line self-absorption corrections (experiments in air).

\begin{tabular}{|c|c|c|c|c|}
\hline Transition & $\lambda(\mathrm{nm})$ & $E_{i}(\mathrm{eV})^{\mathrm{b}}$ & $A_{i j}\left(10^{8} \mathrm{~s}^{-1}\right)^{\mathrm{a}}$ & $\omega_{\mathrm{ref}}(\mathrm{pm}) N_{\mathrm{e}}=10^{17} \mathrm{~cm}^{-3}$ \\
\hline $2 p^{6} 3 p^{2} P_{1 / 2} \rightarrow 2 p^{6} 3 s^{2} S_{1 / 2}$ & 280.270 & 4.422 & 2.57 & $3.9 \pm 5.5 \%(T=14,260)^{\mathrm{c}}$ \\
\hline $2 p^{6} 3 p^{2} P_{3 / 2} \rightarrow 2 p^{6} 3 s^{2} s_{1 / 2}$ & 279.553 & 4.434 & 2.60 & $4.7 \pm 4 \%(T=14,260)^{C}$ \\
\hline $2 p^{6} 3 d^{2} D_{5 / 2} \rightarrow 2 p^{6} 3 p^{2} P_{3 / 2}$ & 279.799 & 8.863 & 4.79 & $16.6 \pm 12 \%(T=30,000)^{\mathrm{d}}$ \\
\hline $2 \mathrm{p}^{6} 3 \mathrm{~d}^{2} \mathrm{D}_{3 / 2} \rightarrow 2 \mathrm{p}^{6} 3 \mathrm{p}^{2} \mathrm{P}_{1 / 2}$ & 270.078 & 8.864 & 4.01 & $16.6 \pm 12 \%(T=30,000)^{\mathrm{d}}$ \\
\hline
\end{tabular}

a Kramida et al., 2014.

b Moore, 1958.

c Goldbach et al., 1982.

d Djeniže et al., 2004.

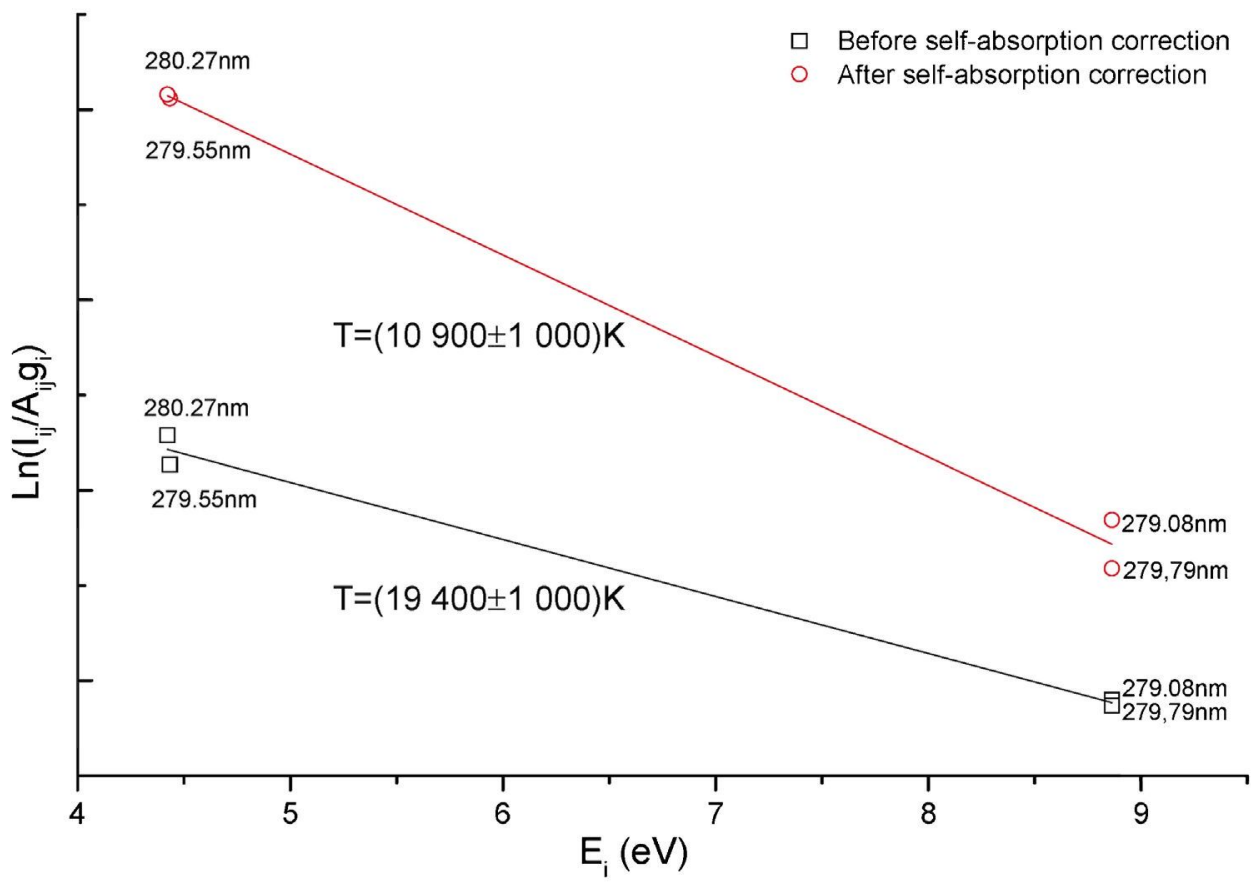

Fig. 10. Boltzmann plot for Mg II spectral lines from laser induced plasma (Al 2024 target) in air. Spectra was recorded at $5 \mu$ s delay time from laser pulse.

McWhirterís criterion (McWhirter, 1965) was used to check the condition for the validity of the LTE. The electron number densities determined in our experiments are well within the range where the LTE conditions are valid, equation:

$N_{\mathrm{e}} \geq 1.6 \times 10^{12} T^{1 / 2}(\Delta E)^{3}$

with $N_{\mathrm{e}}\left(\mathrm{cm}^{-3}\right)$ the electron number density, $T(\mathrm{~K})$ the plasma temperature and $\Delta E(\mathrm{eV})$ the difference in the energy between the upper and lower state of all the investigated transitions.

\section{Conclusion}

A Q-switched laser of Nd:YAG (of 2.5 J per pulse) focused on an aluminum sample (Al2024), in different situations (in air, wet sample and LSP conditions), has been used for the plasma diagnosis produced by laser (LPP). The Stark broadening of the $\mathrm{H} \alpha$-line of Hydrogen ( $656.27 \mathrm{~nm}$ ) has been used to estimate the electron density of plasmas obtained in different conditions and particularly in LSP conditions.

In the case of air, we have tried measured the electron densities with the profile of the $281.62 \mathrm{~nm}$ line of Al II. The results indicate us that this line, in our conditions, presents self-absorption and its usage is inadvisable. In order to make temperature estimation $\mathrm{Mg}$ II in air lines have been used, prior self-absorption quantification.

The measures were realized with different delay times after the laser pulse (1-8 $\mu \mathrm{s}$ ) and with a time window measure of $1 \mu \mathrm{s}$. The electron density obtained in the LSP case was in the range of
$10^{17} \mathrm{~cm}^{-3}$ for the shortest delays until $10^{16} \mathrm{~cm}^{-3}$ for greater delays. We have found, at $5 \mu$ s after the laser pulse, the profile and the position of the $\mathrm{H} \alpha$-line has remained unchanged indicating that at that time the electron density and the electron temperature plasma are identical within the experimental uncertainties. A temperature around $11,000 \mathrm{~K}$ at $5 \mu$ s after delay pulse has been estimated for plasmas in LSP conditions through extrapolation of temperatures in air.

\section{Acknowledgment}

This work has been supported by the Spanish DGI project MAT2012-37782.

\section{References}

Alonso-Medina, A., Colón, C., Herrán-Martínez, C., 2003. Transition from autoionized single-ionized tin states: a theoretical study of the $555 \mathrm{p}\left({ }^{3} \mathrm{p}^{\circ}\right) \mathrm{nl}$ $(\mathrm{nl}=5 \mathrm{~d}, 6 \mathrm{~s})$ levels of Sn II. Astrophys. J. 595, 550-554.

Alonso-Medina, A., Colón, C., 2007. Stark widths of several Pb IIl spectral lines in a laser-induced lead plasma. A\&A 466, 399-402

Alonso-Medina, A., Colón, C., 2008. Measured Stark widths of several Sn I and Sn II spectral lines in a laser induced plasma. Astrophys. J. 672, 1286-1291.

Alonso-Medina, A., 2010. A spectroscopic study of laser-induced tin-lead plasma: transition probabilities for spectral lines of Sn I. Spectrochim. Acta B 65, $158-166$.

Alonso-Medina, A., 2014. Application of laser induced plasma spectroscopy to the measurement of transition probabilities of Ca I. J. Spectrosc. Dinamics 4, 15. 
Ashkenazy, J., Kipper, R., Cane, r M., 1991. Spectroscopic measurements of electron density of capillary plasma based on Stark broadening of hydrogen lines. Phys. Rev., A 43 (10), 5568-5574.

Bogen, P., Lie, Y.T., 1980. Detection of atomic hydrogen by resonance fluorescence using two-step excitation with L $\alpha$ and H $\alpha$ radiation. J. Nucl. Mater. 93-94, $363-367$.

Berthe, L., Fabbro, R., Peyre, P., Tollier, L., Bartnicki, E., 1997. Shock waves from a water-confined laser-generated plasma. J. Appl. Phys. 82 (6), 2826-2833.

Colón, C., Hatem, G., Verdugo, E., Ruiz, P., Campos, J., 1993. Measurement of the Stark broadening and shift parameters for several ultraviolet lines of singly ionized aluminum. J. Appl. Phys. 73 (10), 4752-4758.

Colón, C., Alonso-Medina, A., Herrán-Martínez, C., 1999. Spectroscopic study of a laser-produced lead plasma: experimental atomic transitions probabilities for Pb III lines. J. Phys. B At. Mol. Opt. Phys, 32, 3887-3897.

Colón, C., Alonso-Medina, A., 2006. Application of a laser produced plasma: experimental Stark widths of single ionized lead lines. Spectrochim. Acta B 61 , $856-863$.

Cremers, A.D., Chinni, R.C., 2009. Laser-induced breakdown spect roscopy-capabilities and limitations. App. Spectrosc. Rev. 44, 457-506.

De Giacomo, A., Dell'Aglio, M., Colao, F., Fantoni, R., 2004. Double pulse laser produced plasma on metallic target in sea water basic aspects and analytical approach. Spectrochim. Acta Part B 59, 1431-1438.

De Giacomo, A., Dell'Aglio, M., Gaudiuso, R., Cristoforetti, G., Legnaioli, S., Palleschi, V., Tognoni, E., 2008. Spatial distribution of hydrogen and other emitters in aluminum laser-induced plasma in air and consequences on spatially integrated laser-induced breakdown spectroscopy measurements. Spectrochim. Acta B 6 (3), 980-987.

Djeniže, S., Bukvić, S., Srećković, A., Platiša, M., 2004. Mg II spectral line broadening in helium, oxygen and argon-helium plasmas. A\&A 424, 561-564.

El Sherbini, A., El Sherbini, M., ThM. Heagzy, H., Cristoforetti, G., Legnaioli, S., Palleschi, V., Pardini, L., Salvetti, A., Tognoni, E., 2005. Evaluation of self-absorption of aluminum emission lines in laser-induced breakdown spectroscopy measurements. Spectrochim. Acta B 60, 1573-1579.

El Sherbini, A.M., Heagzy, H., El Sherbini, Th. M., 2006. Measurement of electron density utilizing the $\mathrm{H} \alpha$-line from laser produced plasma in air. Spectrochim. Acta B 61, 532-539.

El Sherbini, A.M., Al Amer, A.A.S., Hassan, A.T., El Sherbini, Th. M., 2012. Measurements of plasma electron temperature utilizing magnesium lines appeared in laser produced aluminum plasma in air. Optics Photonic J. 2, $278-285$.

Escarguel, A., Ferha, B., Lesage, A., Richou, J., 2000. A single laser spark in aqueous medium. J. Quant. Spectrosc. Radiat. Transfer 64, 353-361.

Fabbro, R., Foumier, J., Ballard, P., Devaux, D., Virmont, J., 1990. Physical study of laser-produced plasma in confined geometry. J. Appl. Phys. 68 (2), 775-784.

Fairand, B.P., Wilcox, B.A., Gallagher, W.J., Willians, D.N., 1972. Laser shock-induced microstructural and mechanical property changes in 7075 aluminum. J. Appl. Phys. 43, 3893-3895.

Fairand, B.P., Clauer, A.H., 1979. Laser generation of high-amplitude stress waves in materials. J. Appl. Phys. 50, 1497-1502
Goldbach, C, Nollez, G, Plomdeur, P., Zimmermann, J.P., 1982. Stark-width measurements of neutral and singly ionized magnesium resonance lines in a wall-stabilized arc. Phys, Rev. A 25, 2596-2605.

Griem, H.R., 1974. Spectral Line Broadening by Plasmas. Academic Press, New York.

Griem, H.R., 1997. Principles of Plasma Spectroscopy. Cambridge University Press, United Kingdom.

Kepple, P., Griem, H.R., 1968. Improved Stark profile calculations for the hydrogen lines $\mathrm{H}_{\alpha}, \mathrm{H}_{\beta}, \mathrm{H} \gamma$ and $\mathrm{H}_{\delta}$. Phys. Rev. 173, 317-325.

Konjevic, N., 1999. Plasma broadening and shifting of non hidrogenic spectral line: present status and applications. Phys. Rep. 316, 339-401.

Kramida, A., Ralchenko, Y., Reader, J., 2014. NIST ASD team. In: NIST Atomic Spectra Database (ver. 5.2). National Institute of Standards and Technology, Gaithersburg, MD [Online Available 2015, January 15] http://physics.nist.gov/ asd.

Lochte-Holtgreven, W., 1968. Plasma Diagnostics. North-Holland Publishing Company, Amsterdam.

Lyon, S.P., Johnson, J.D., 1992. SESAME: The Los Alamos National Laboratory Equation of State Database, Technical Report, LA-UR-92-3407. Los Alamos National Laboratory, Los Alamos NM.

McWhirter, R.W.P., 1965. In: Huddlestone, R.H., Leonard, S.L. (Eds.), Plasma Diagnostic Thechniques. Academic, New York, Chapter 5.

Moore, C.E., 1958. Atomic energy levels. In: NBS 467. U. S. GPO, Washington DC

Morales, M., Ocaña, J.L., Molpeceres, C., Porro, J.A., García-Beltrăn, A., 2008. Mode based optimization criteria for the generation of deep compressive residual stress fields in high elastic limit metallic alloys by ns-laser shock processing. Surf. Coat. Tech. 202, 2257-2262

Musazzi, S., Perini, U., 2014. Laser Induced Breakdown Spectroscopy Theory and Applications. Springer-Verlag, Berlîn.

Noll, R., Sturm, V., Aydin, Ü., Eilers, D., Gehlen, C., Höhne, M., Lamott, A., Makowe, J. Vrenegor, J., 2008. Laser-induced breakdown spectroscopy-from research to industry, new frontiers for process control. Spectrochim. Acta B 63, 1159-1166.

Noll, R., 2012. Laser-induced Breakdown Spectroscopy. Springer-Verlag, Berlin Heidelberg.

Ocaña, J.L., Molpeceres, C., Morales, M., García-Beltrán, A., 2000. Model for the coupled predictive assessment of plasma expansion and material compression in laser shock processing applications high-power laser ablation. II Proc. SPIE $3885,252-265$

Ocaña, J.L., Molpeceres, C., Porro, J.A., Gômez, G., Morales, M., 2004. Experimental assessment of the influence of irradiation parameters on surface de formation and residual stresses in laser shock processed metallic alloys. Appl. Surf. Sci. $238,501-505$.

Radziemski, L.J., Cremers, D.A., 1989. Laser-induced Plasma and Applications. Dekker, New York.

Sano, Y., Mukai, N., Okazaki, K., Obata, M., 1997. Residual stress improvement in metal surface by underwater laser irradiation. Nucl. Instrum. Methods Phys. Res, B $121,432-436$

Wu, B., Shin, C., 2005. A self-closed thermal model for laser shock peening under the water confinement regime configuration and comparisons to experiments. J. App. Phys. 97, 113517. 